\title{
Fortalecimento da musculatura expiratória na esclerose múltipla: relato de caso
}

Strengthening muscle expiratory in

multiple sclerosis: case report

FisiSenectus . Unochapecó Ano 3, n. 2 - Jul/Dez. 2015 p. 25-32

Carine Caldatto.nini_caldatto@hotmail.com

Fisioterapeuta pela Universidade Regional Integrada do Alto Uruguai e das Missões - URI Erechim.

Fernanda Dal'Maso Camera. ferdalmaso@hotmail.com

Fisioterapeuta, Mestre, Docente do Departamento de Ciências da Saúde da Universidade Regional Integrada do Alto Uruguai e das Missões - URI Erechim.

Elvis Wisniewski.04.elvis@gmail.com

Fisioterapeuta, Mestre, Docente do Departamento de Ciências da Saúde da Universidade Regional Integrada do Alto Uruguai e das Missões - URI Erechim.

Márcia Bairros de Castro.mbairros@uricer.edu.br

Fisioterapeuta, Mestre, Docente do Departamento de Ciências da Saúde da Universidade Regional Integrada do Alto Uruguai e das Missões - URI Erechim.

Miriam Salete Wilk Wisniewski. miriamww@gmail.com

Fisioterapeuta, Doutora, Docente do Departamento de Ciências da Saúde da Universidade Regional Integrada do Alto Uruguai e das Missões - URI Erechim.

\section{Resumo}

Introdução: a esclerose múltipla é uma doença crônica do sistema nervoso central, caracterizada por múltiplas áreas de inflamação, desmielinização e formação de cicatrizes gliais na substância branca. A diminuição no volume pulmonar, na capacidade vital, na resistência respiratória e na eficiência do mecanismo da tosse estão entre as principais causas de morbimortalidade. Objetivos: verificar os efeitos do fortalecimento muscular expiratório em um indivíduo com diagnóstico de esclerose múltipla do tipo remitente-recorrente.Materiais e métodos: a avaliação contou com anamnese, seguida dos testes de manovacuometria, espirometria e pico de fluxo expiratório. A intervenção deu-se em uma frequência semanal ( $2 x /$ sem), durante dez semanas, utilizando o aparelho Threshold PEP $®$. 0 treinamento foi realizado em seis séries de dez exercícios. A reavaliação ocorreu em dois momentos, imediatamente após o término das vinte intervenções e após trinta dias da mesma. Resultados: o treinamento muscular expiratório proporcionou melhora da condição respiratória, indicando a importância da implementação dessa terapia, de forma precoce, na esclerose múltipla.

\section{Palavras-chave}

Esclerose Múltipla. Exercícios respiratórios. Fisioterapia. 


\begin{abstract}
Introduction: multiple sclerosis is a chronic disease of the central nervous system, wich is characterized by multiple areas of inflammation, demyelination and formation of glial scars in the white substance. Decrease in lung volume, vital capacity, respiratory resistance and efficiency of the cough engine are among the leading causes of morbidity and mortality. Objective: to verify the effects of expiratory muscle strength in an individual diagnosed with multiple sclerosis relapsing-remitting type. Materials and methods: the evaluation included history, followed by the manometer tests, spirometry and peak expiratory flow. The intervention was given on a weekly frequency, 2 times a week, for ten weeks using Threshold PEP $\AA$ device. The training was conducted in six ten exercises series. The revaluation took place in two stages, immediately after the end of the twentieth interventions and after thirty days thereof. Results: there was $45 \%$ increase in maximal inspiratory pressure, $60 \%$ of the maximal expiratory pressure after 30 days of intervention, and peak expiratory flow showed up with value about $30 \%$ higher compared to initial evaluation. Conclusion: the expiratory muscle training provided an increase in respiratory muscle strength, demonstrating significant importance of implementing this type of therapy in multiple sclerosis earlier.
\end{abstract}

Keywords: Multiple Sclerosis. Breathing Exercises. Physical Therapy Specialty.

\section{Introdução}

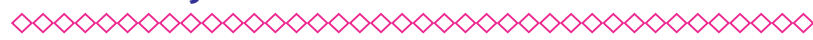

A Esclerose Múltipla (EM) é uma doença autoimune, desmielinizanteinflamatóriacrônicado sistema nervoso central (SNC), neurodegenerativa e progressiva, com evolução imprevisivel ${ }^{1,2}$. Afeta principalmente mulheres jovens com idade entre 20 a 40 anos, em uma proporção de 3:13. É considerada a segunda causa mais comum de incapacidade em adultos jovens ${ }^{4}$, tendo como principais sintomas a perda aguda da visão (neurite óptica) e da força muscular, alterações na coordenação motora e na sensibilidade, disfunção cognitiva e fadiga, que podem apresentar-se isolada ou associadamente ${ }^{5}$. Esses sintomas podem constituir a forma "remitente-recorrente" da doença, que compreende $85 \%$ dos $\operatorname{casos}^{3}$.

A fisiopatologia caracteriza-se por lesões na substância branca do SNC, com perda de mielina, axônios neuronais e oligodendrócitos. A destruição de oligodendrócitos produz áreas de desmielinização, denominadas placas, na substância branca do SNC, causando lentidão e bloqueio da transmissão de sinais ${ }^{6}$.

Complicações respiratórias representam as principais causas de morbimortalidade na EM. Diversos estudos demonstram que aproximadamente $50 \%$ dos indivíduos com EM vão a óbito em decorrência dessas complicações, sendo a pneumonia, a causa mais prevalente $\mathrm{e}^{7-11}$. A paresia dos músculos expiratórios é comum, sendo os abdominais primariamente acometidos, seguidos da musculatura intercostal e, por fim, o diafragma ${ }^{12}$. 0 comprometimento desses músculos, ocasiona uma inabilidade para a geração da tosse, que associada a outras alterações, como diminuição no volume pulmonar, na capacidade vital e na resistência respiratória, são reconhecidas como fatores predisponentes às infecções respiratórias.

Frente a essa breve contextualização, foram objetivos deste estudo: verificar os efeitos de um programa de fortalecimento muscular expiratório em indivíduos com EM, mensurar a força muscular respiratória, o pico de fluxo expiratório e as capacidades pulmonares antes e após o programa de fortalecimento expiratório.

\section{Materiais e métodos}

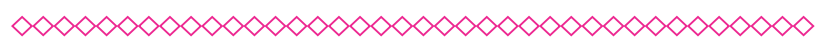

0 presente estudo caracterizou-se como relato de caso, de caráter longitudinal, não controlado, do tipo intervenção, com abordagem quantitativa. Foi realizado com um indivíduo do sexo feminino, de 30 anos de idade, com esclerose múltipla remitente-recorrente (EMRR), diagnosticada no ano de 2006. 
A avaliação inicial contou com a anamnese seguida de exame físico, com intuito de avaliar a força muscular respiratória, o pico de fluxo expiratório e a capacidade pulmonar da voluntária. A força muscular respiratória foi avaliada por meio do teste de Manovacuometria, utilizando - Manovacuômetro digital MVD300, portátil, marca GlobalMed, a fim de identificar a pressão inspiratória máxima (PImáx) a partir do volume residual (VR) e a pressão expiratória máxima (PEmáx) a partir da capacidade pulmonar total $(\mathrm{CPT})^{13}$. 0 pico de fluxo expiratório foi avaliado por meio do teste de Peak Flow, ${ }^{14}$ utilizando o medidor de fluxo portátil da marca Peak Flow Meter ${ }^{\circledR}$. Foram coletadas cinco expirações forçadas, intervaladas por um minuto entre elas, considerando-se 0 maior valor obtido dentre as manobras aceitáveis (máximas) e reprodutíveis (diferença de 10\% ou menos entre os valores aferidos). A espirometria ${ }^{15}$ foi obtida por meio do Espirômetro Spirolab II, considerando-se a CVF, a relação VEF1/CVF e VEF1 como parâmetros de interesse.

Paralelamente avaliou-se o grau de fadiga a partir da escala de severidade de fadiga, composta por nove itens, com pontuação variando entre 1 a 7, totalizando escores entre 9 e 63. Considerase o escore maior ou igual a 28 como indicativo de fadiga. Esse teste foi aplicado diariamente, previamente e imediatamente após cada intervenção, visando identificar seu grau de fadiga, considerada impeditiva caso fossem identificados valores iguais ou superiores a $28^{16,17}$.

A intervenção fisioterapêutica foi baseada no incremento de carga para melhora da força dos músculos expiratórios. Para esse treinamento utilizou-se 0 aparelho muscular respiratório Threshold PEP $\circledast$ da marca Respironics. O valor inicial correspondeu a 30\% da PEmáx obtida na avaliação inicial, sendo reajustada a cada semana. 0 treinamento muscular foi realizado durante dez semanas, $2 x /$ sem, $1 / x /$ dia, por aproximadamente 30 minutos, totalizando 20 atendimentos. A voluntária iniciou os exercícios com carga de 9 $\mathrm{cm} / \mathrm{H} 2 \mathrm{O}$ e terminou com carga de $15 \mathrm{~cm} / \mathrm{H} 2 \mathrm{O} .0$ treinamento foi realizado em seis séries de dez exercícios com intervalo de um minuto entre as séries, sendo esse protocolo elaborado pelos autores.
A reavaliação foi realizada imediatamente após o término da intervenção e 30 dias após a mesma, utilizando-se os mesmos instrumentos de pesquisa iniciais. Os dados foram analisados por meio de estatística descritiva simples, sendo que estes foram comparados com os valores preditos na literatura.

Para a realização deste estudo, foram seguidas as diretrizes e normas regulamentadoras da Resolução 466/2012 do Conselho Nacional de Saúde do Ministério da Saúde que trata de pesquisa envolvendo seres humanos. 0 estudo foi previamente submetido ao Comitê de Ética em Pesquisa da Universidade Regional Integrada do Alto Uruguai e das Missões URI Erechim e foi aprovado por meio do CAAE: 24172313.5.0000.5351 e Parecer no 488.740 em 29 de novembro de 2013.

\section{Resultados}

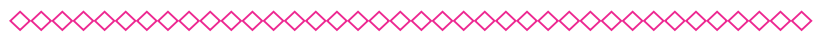

Ao exame físico, a voluntária, cadeirante, apresentou-se consciente, contactuante, com padrão respiratório misto, com predomínio diagragmático, eupneica, sem uso de musculatura acessória, com abdômen globoso, tórax normal e expansibilidade torácica simétrica. Sinais vitais: FC: 78 bpm, FR: 17 rpm; PA: 110/80 mmHg; peso: $75 \mathrm{~kg}$, altura: $1,71 \mathrm{~cm}$. 0 Gráfico I demonstra os resultados referentes à força muscular respiratória obtida por meio da manovacuometria em três momentos.

Analisando os resultados do Gráfico I, é possível afirmar que, ao início da intervenção, a voluntária apresentava fraqueza muscular, tanto em musculatura inspiratória como expiratória. Observa-se que os valores alcançados tanto em PImáx como em PEmáx são inferiores ao previsto para sua faixa etária, que correspondem respectivamente a $(-91,5 \pm 10,1)$ e $(100,6 \pm 12,1)$.

Seguindo-se a apresentação dos dados, o Gráfico II apresenta os valores obtidos a partir do teste do pico de fluxo expiratório, mensurado nos mesmos três momentos, no qual o maior valor obtido foi na reavaliação. Analisando-se o Gráfico II, observa-se que os valores do pico de fluxo expiratório obtidos na avaliação, reavaliação 
imediata e 30 dias após, encontram-se $46,88 \%$ e $60,27 \%$, abaixo dos valores previstos para a idade, gênero e altura da voluntária deste estudo.

A avaliação da função pulmonar por meio da Espirometria, nas três avaliações, apresentou valores que podem ser analisados na Tabela I. A partir dos valores apresentados acima, verificouse não haver presença de distúrbios ventilatórios, uma vez que os valores encontram-se acima do previsto. Foi possível observar também que com o treinamento respiratório ocorreu uma melhora gradativa da capacidade vital forçada (CVF).

\section{Discussão}

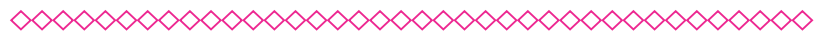

Observou-se que após vinte intervenções a condição de força muscular da voluntária deste estudo permaneceu nos valores que conceituam a fraqueza muscular. No entanto, a PImáx aproximou-se dos valores normais e após trinta dias permaneceu levemente superior ao valor inicial, atingindo 56,8\% do valor predito. Com relação à PEmáx, pode-se dizer que a força muscular expiratória, na avaliação, alcançava 30,8\% do previsto e após 30 dias 48,7\%, sugerindo que ambas as pressões responderam favoravelmente ao treinamento aqui proposto.

0 estudo do comportamento da força muscular inspiratória, sob treinamento domiciliar durante dez semanas, com 42 indivíduos com EM, proporcionou $81 \%$ de aumento da força muscular inspiratória ao final do treinamento, 0 que representa redução do comprometimento da musculatura inspiratória ${ }^{19}$. De modo semelhante, outro estudo ${ }^{20}$ analisou os resultados do treinamento muscular inspiratório realizado em três séries de dez repetições de inspiração resistida, duas vezes/dia, em dias alternados, durante dez semanas. Nesse estudo, após o treinamento, os 15 voluntários com EM que completaram o programa apresentaram aumento significativo em ambas as pressões, PImáx e PEmáx, mantidas um mês após a conclusão do protocolo.

O treinamento da musculatura expiratória foi analisado em 22 indivíduos com EM avançada e demonstrou aumento de força muscular inspiratória e expiratória ${ }^{21}$. Estudos comparativos ${ }^{22}$ da função respiratória entre indivíduos com EM nos estágios iniciais e os valores esperados a partir da população saudável observaram uma redução na média da PImáx em 23\% do valor estimado, em comparação com $40 \%$ na PEmáx.

Os efeitos do treino respiratório também foram verificados em oito indivíduos com EM que apresentavam fraqueza muscular respiratória ${ }^{23}$. A amostra apresentou média de 53 anos de idade e foi submetida ao treinamento de resistência ao fluxo aéreo, duas vezes/dia, cinco dias/semana, por quatro semanas. Houve $31 \%$ de aumento na PImáx, $31 \%$ na PEmáx e $21 \%$ no pico de fluxo, demostrando melhora da força muscular respiratória e da capacidade ventilatória. Esses resultados corroboram com o observado em nosso estudo, no qual o treinamento muscular expiratório proporcionou o aumento da força muscular respiratória, visto que na reavaliação a PImáx apresentou valor de aproximadamente $45 \%$ superior ao obtido na avaliação; a PEmáx, após 30 dias da intervenção, registrou valor $60 \%$ superior ao obtido na avaliação; e o pico de fluxo na reavaliação apresentou valor aproximadamente $30 \%$ superior ao inicial.

A anamnese realizada com a voluntária do presente estudo indicou ausência de dispneia. Este é um sinal raramente presente na $\mathrm{EM}^{11}$, pois conforme a progressão neurológicodegenerativa, haverá incapacidade motora grave e comprometimento significativo da força muscular respiratória, possivelmente relacionada a atividades motoras restritas e a uma maior disfunção muscular expiratória do que inspiratória. Isso é explicado pelo fato de que a fraqueza na EM ascende lentamente das extremidades inferiores para as extremidades superiores (musculatura expiratória é afetada antes da inspiratória). Uma vez que o diafragma é inervado pelo nervo frênico, este será o último músculo respiratório a ser afetado, o que desperta a importância da implantação de programas de fortalecimento muscular expiratório precoce nessa doença.

Na posição sentada, a atividade tônica da musculatura abdominal promove uma facilitação direta na ação diafragmática, evitando o 
encurtamento excessivo durante a inspiração. A alta complacência da cavidade abdominal proporcionada pela fraqueza da musculatura abdominal, como a que ocorre na maioria dos pacientes neurológicos, mantém a zona de aposição do diafragma mais baixa, assim a sua efetividade diminui ${ }^{24}$. Acredita-se que no presente estudo, o treinamento muscular expiratório tenha promovido o aumento da força da musculatura abdominal, repercutindo no aumento da PEmáx mesmo após o término do treinamento. Da mesma forma, a PImáx, após 30 dias, permaneceu levemente superior ao valor obtido inicialmente. Isto é, mesmo não alterando a condição de fraqueza muscular, o treinamento proporcionou aumento nas pressões, o que sugere a importância do treinamento muscular expiratório continuado na EM.

No tocante aos resultados espirométricos, o presente estudo demonstrou não haver alteração na relação VEF1/CVF tanto antes como após a intervenção. Estudo comparativo ${ }^{25}$ utilizando como instrumentos de pesquisa o exame clínico neurológico, a espirometria e a pressão respiratória máxima de 52 indivíduos, sendo 27 com EMRR e 25 saudáveis, demonstrou não haver diferenças estatisticamente significativas quanto à idade, ao sexo, à altura, ao peso e ao índice de massa corporal entre os grupos. Quanto à espirometria, a média dos valores para a capacidade pulmonar total, VEF1 e VEF1/CVF, foram normais em ambos os grupos. 15 pacientes com EMRR exibiram uma redução na pressão expiratória máxima, porém somente um paciente apresentou redução da pressão inspiratória máxima. Os resultados desse estudo corroboram parcialmente com os obtidos em nossa pesquisa.

\section{Considerações finais}

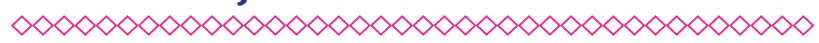

Os resultados demonstraram que o treinamento muscular expiratório proporcionou ganhos significativos na condição respiratória da voluntária deste estudo. Logo, treinar o músculo expiratório de forma precoce na esclerose múltipla é fundamental para evitar complicações respiratórias.

\section{Referências}

$\alpha_{\infty}<\infty<\infty<\infty<\infty<\infty<\infty<\infty<\infty<\infty<\infty<\infty<\infty<\infty<\infty<\infty$

1. Sospedra M, Martin R. Immunology of multiple sclerosis. Annu. Rev. Immunol. 2005; 23: 683-747.

2. Lassmann H. Multiple sclerosis: lessons from molecular neuropathology. Exp. Neurol. 2014; 262:2-7.

3. Noseworthy JH, Lucchinetti C, Rodriguez M, Weinshenker BG. Multiple sclerosis. N Engl J Med. 2000 Sep 28; 343(13):938-52.

4. Compston A, Coles A. Multiple sclerosis. Lancet. 2002 Apr 6; 359(9313):1221-31.

5. Calabresi PA. Diagnosis and management of multiple sclerosis. Am. Fam. Physician. 2004 Nov 15; 70 (10):1935-44.

6. Martino G, Adorini L, Rieckmann P, Hillert J, Kallmann B, Comi G, et al. Inflammation in multiple sclerosis: the good, the bad, and the complex. Lancet Neurol. 2002 Dec;1 (8):499-509.

7. Sadovnick AD, Eisen K, Patty DW, Ebers GC. Cause of death in patients attendingmultiple sclerosis clinics. Neurology. 1991 Aug; 41(8): 1193-6.

8. Foglio K, Clini E, Facchetti D, Vitacca M, Marangoni S, Bonomelli M, et al. Respiratory muscle function and exercise capacity in Multiple Sclerosis. Eur. Respir. J. 1994; 7:23-8.

9. Smeltzer SC, Utell MJ, Rudick RA, Herndon RM. Pulmonary function and dysfunction in multiple sclerosis. Arch. Neurol. 1998 Nov; 45(11):1245-9.

10. Gosselink R, Kovacs L, Decramer M. Respiratory muscle involvement in multiple sclerosis. Eur. Respir. J. 1999 Feb; 13(2):449-54.

11. Buyse B, Demedts M, Meekers J, Vandergaer $L$, Rochette F, Kerkhofs $L$. Respiratory dysfuntion in Multiple Sclerosis: a prospective analysis of 60 patients. Eur. Respir. J. 1997 Jan; 10 (1):139-45.

12. Smeltzer SC, Lavietes MD, Cook SD. Exporatory training in multiple sclerosis. Arch. Phys. Med.

Rehabil. 1996 Sep; 77(9):909-12. 
13. Neder JA, Andreoni S, Lerario MC, Nery LE. Reference values for lung function tests. II. Maximal respiratory pressures and voluntary ventilation. Braz. J. Med. Biol. Res. 1999; 32(6):719-27.

14. Pereira CAC. Espirometria. J. Pneumol. Out 2002 28(Supl 3):1-82.

15. Costa D. Fisioterapia respiratória básica. São Paulo: Atheneu; 2004.

16. Neuberger GB. Measures of fadigue. Arthritis Rheum. 2003 Out; 49(S5):175-83.

17. Tilbery CP. Esclerose Múltipla no Brasil aspectos clínicos e terapêuticos. São Paulo: Atheneu; 2005.

18. Leiner GC, Abramowitz S, Small MJ, Stenby VB, Lewis WA. Expiratory peak flow rate. Standard values for normal subjects. Use as a clinical test of ventilatory function. Am. Rev. Respir. Dis. 1963 Nov; 88: 644-51.

19. Fry DK, Pfalzer LA, Chokshi AR, Wagner MT, Jackson ES. Randomized control trial of effects of a 10-week inspiratory muscle training program on measures of pulmonary function in persons with multiple sclerosis. J. Neurol. Phys. Ther. 2007; 31(4):162-72.

20. Klefbeck B, Hamrah Nedjad J. Effect of inspiratory muscle training in patients with multiple sclerosis. Arch. Phys. Med. Rehabil. 2003 Jul; 84 (7):994-9.

21. Gosselink R, Kovacs L, Ketelaer P, Carton $H$, Decramer M. Respiratory muscle weakness and respiratory muscle training in severely disabled multiple sclerosis patients. Arch. Phys. Med. Rehabil. 2000 Jun; 81(6):747-51.

22. Mutluay FK, Gürses HN, Saip S. Effects of multiple sclerosis on respiratory functions. Clin. Rehabil. 2005 Jun; 19(4):426-32.

23. Olgiati R, Burgunder J-M, Mumenthaler $M$. Increased energy cost of walking in multiple sclerosis effect of spasticity, ataxia and weakness. Arch. Phys. Med. Reabil. 1988; 69: 846-9.
24. Machado MGR. Bases da Fisioterapia Respiratória: Terapia Intensiva e Reabilitação. Rio de Janeiro: Guanabara Koogan; 2008.

25. Carvalho SR, Alvarenga Filho H, PapaisAlvarenga RM, Chacur FH, Dias RM. Is it useful to perform carbon monoxide diffusion capacity and respiratory muscle function tests in patients with multiple sclerosis without disability? Respirology. 2012 Jul 17(5):869-75. 


\section{Anexos}

Gráfico I. Valores de PImáx e PEmáx, obtidos na avaliação, reavaliação e após trinta dias do término da intervenção, em comparação aos valores preditos.

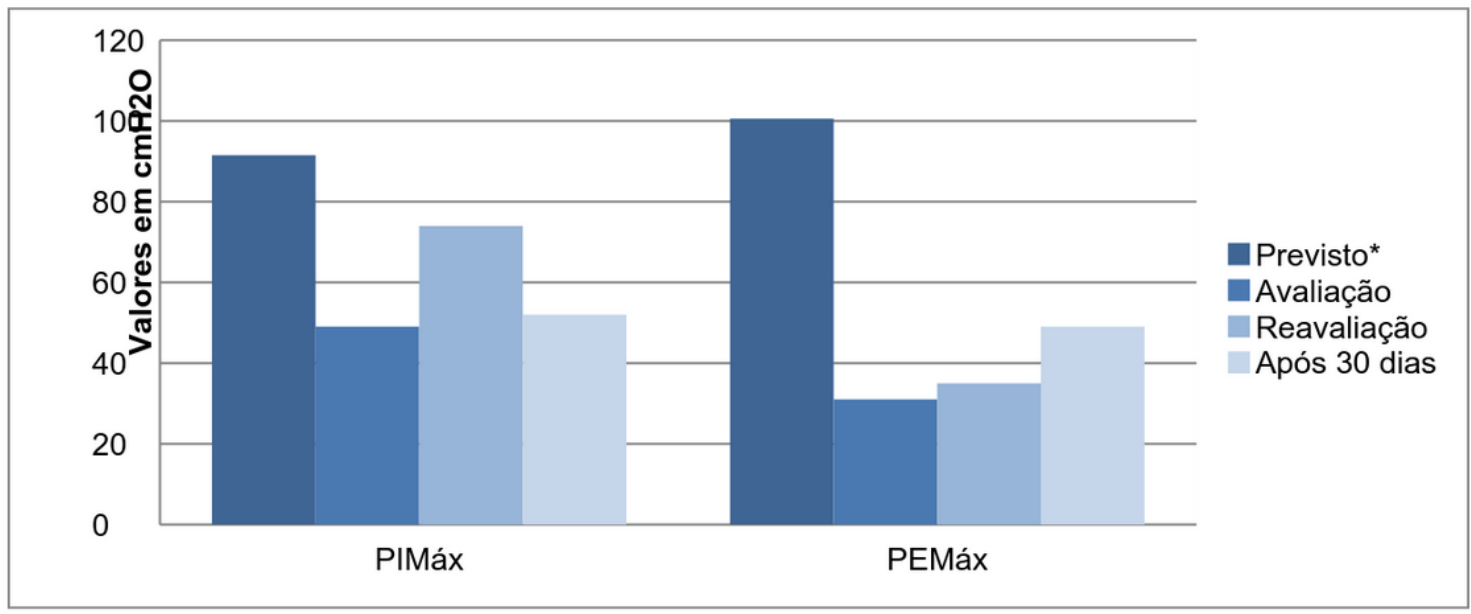

*Valores previstos conforme Neder et al., (1999).

(clique para voltar ao texto)

Gráfico II. Valores obtidos através do teste do pico de fluxo expiratório, na avaliação, reavaliação e após 30 dias da intervenção.

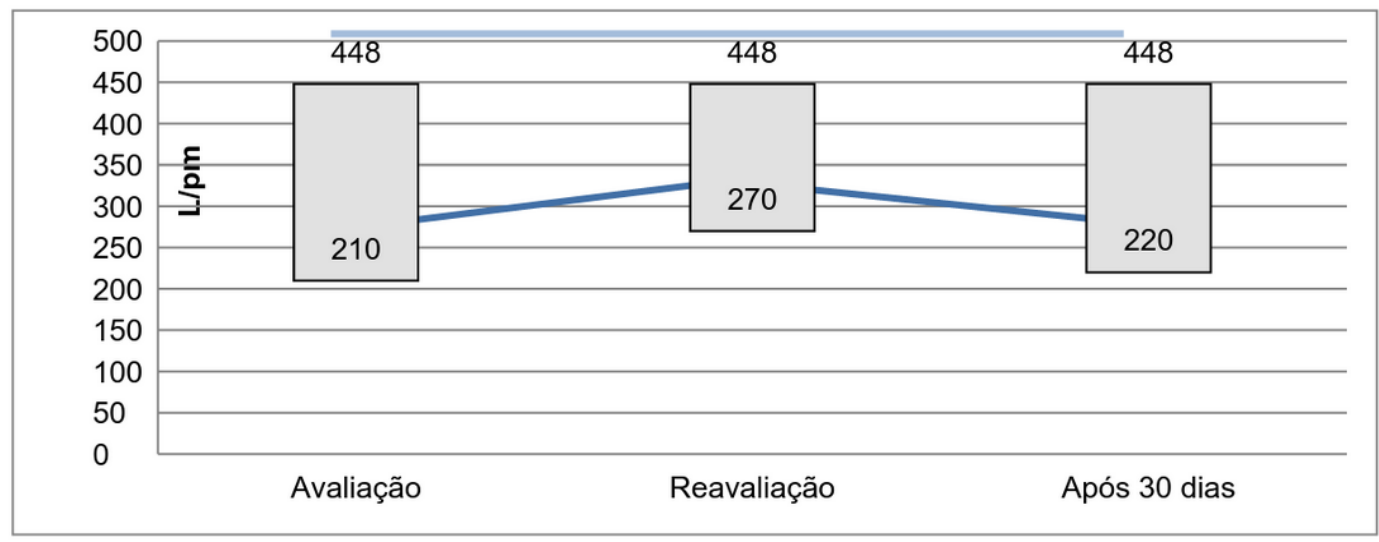

*Valores preditos conforme Leiner (1963). 
Tabela I. Valores referentes à espirometria durante a avaliação, reavaliação e após 30 dias da intervenção e comparados com os valores preditos.

\section{Espirometria}

\begin{tabular}{l|l|l|l|l}
\hline & $\begin{array}{l}\text { CVF } \\
\text { Medida }\end{array}$ & $\begin{array}{l}\text { CVF } \\
\text { Prevista }\end{array}$ & $\begin{array}{l}\text { VFE1/CVF\% } \\
\text { Medida }\end{array}$ & $\begin{array}{l}\text { VFE1/CVF\% } \\
\text { Prevista }\end{array}$ \\
\hline Avaliação & $\mathbf{3 . 1 9 ( 8 0 )}$ & 4.01 & $\mathbf{9 4 . 4 ( 1 1 3 \% )}$ & 83.8 \\
\hline Reavaliação & $\mathbf{3 . 3 1 ( 8 3 )}$ & 4.01 & $\mathbf{9 2 . 1 ( 1 1 0 \% )}$ & 83.8 \\
\hline Após 30 dias & $\mathbf{3 . 5 7}(\mathbf{8 9})$ & 3.99 & $\mathbf{8 8 . 2 ( 1 0 6 \% )}$ & 83.6
\end{tabular}

CVF: Capacidade Vital Forçada. VFE1/CVF: Relação entre o Volume Expiratório Forçado no Primeiro segundo e a Capacidade Vital Forçada. 